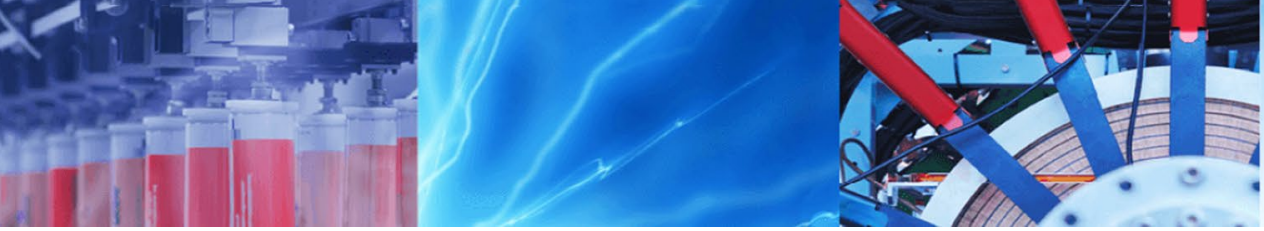

Research Article

\title{
Methodology for the formation of photoresist films with uniform thicknesses of several hundred micrometers
}

\author{
Tamio Fujiwara' ${ }^{1} \cdot$ Haruyuki Kinoshita ${ }^{2} \cdot$ Hiroshi Ohue $^{1} \cdot$ Teruo Fujii $^{2}$
}

Received: 9 August 2020 / Accepted: 19 October 2020 / Published online: 27 October 2020

(c) Springer Nature Switzerland AG 2020

\begin{abstract}
To enhance the capabilities of photolithography in the field of microfluidic technology, this study establishes a method to produce a photoresist film with a uniform thickness spanning several hundred micrometers. Herein, the target thickness of the trial production films is $400 \mu \mathrm{m}$. The SU-8 3000 series and 4-inch silicon wafers were adopted as the photoresist and substrates, respectively. It was found that when a large amount of SU-8 is formed using the normal procedure, and the film thickness is biased in a certain direction and becomes non-uniform. The reason for this film thickness bias has not been clarified, but it is confirmed that the bias is induced during the soft baking of the resist and not during the coating process. Accordingly, this study proposes a new method to remediate and equalize the biased films. Before film remediation, the photoresist should be adequately soft-baked. However, it is difficult to do this inside of the thick film by simple heating. Thus, some techniques used to facilitate baking thick resist layers are also introduced. Trial production films that are appropriately remediated are obtained. It is shown that in the center region of a remedied film with a 40-mm diameter, the deviation of the local thickness is within 1\%, i.e., the thickness bias is completely eliminated. The thickness can also be controlled by remediation with a reproducibility of $1 \%$. The results indicate that the proposed method used to form thick photoresist films is effective for practical applications.
\end{abstract}

Keywords Photolithography $\cdot$ Thick film $\cdot$ Photoresist $\cdot$ SU-8 $\cdot$ Precise formation

\section{Introduction}

Microfluidic technology often employs microchannels made of elastomers, such as polydimethylsiloxane (PDMS) that are cast with mold masters using soft lithographic techniques [1-6]. As a method used to make molds, photolithography has several advantages related to geometry, surface roughness, size precision, etc., when compared with other methods including ordinary machining. In the case of microchannels with rectangular cross-sectional areas, the molds used to cast the channels are typically fabricated from the photoresist formed on the substrate using photolithography. The shape of the formed resist is copied to the channel of the cast elastomer, so that the depth of the replica channel is equivalent to the formed resist film thickness, which should be adjusted to match the required channel depth. The depths of the microchannels required in microfluidic technologies frequently span several hundred micrometers [7-9]. Thus, films with equivalent thicknesses are required to cast these types of channels. However, as the available thickness range of general commercial photoresists does not exceed approximately $100 \mu \mathrm{m}$, there is no commercial product for such thicker films.

One type of photoresist appropriate for thick films is SU-8 (MicroChem Corp.) [10, 11]. In many studies, SU-8

$\triangle$ Tamio Fujiwara, tfujiwa@tcu.ac.jp | 1 Department of Mechanical Engineering, Faculty of Science and Engineering, Tokyo City University, 1-28-1 Tamazutsumi, Setagaya-ku, Tokyo 158-8557, Japan. ${ }^{2}$ Institute of Industrial Science, The University of Tokyo, 4-6-1 Komaba, Meguro-ku, Tokyo 153-8505, Japan. 
has been used for micro-fabrication because of its superior properties [12-16]. The SU-8 manufacturer provides a "recipe" for the appropriate procedure to form SU-8 films with thicknesses within approximately one hundred and a couple of tens of micrometers. Du et al. [17] studied the adhesion strength between the substrate and "thick" SU-8 film with a thickness of $120 \mu \mathrm{m}$. Mao et al. [18] proposed a method for releasing SU-8 structure from the substrate; the maximum thickness of the structures they experimented with was $150 \mu \mathrm{m}$.

In a typical SU-8 formation process, a soft-baked resist film applied on a substrate crosslinks due to exposure to ultraviolet (UV) rays and the geometry of the formed resist is controlled by the pattern of the photomask used when the exposure. In the case that the resist thickness is more than one hundred and some tens $\mu \mathrm{m}$, a large gap is created between the resist and the photomask during exposure due to the large non-uniformity in film thickness after soft baking, making it impossible to expose the resist in an accurate pattern [19]. Liu et al. and Amato et al. [20, 21] investigated a method to create high-aspect-ratio microstructures using thick SU-8 films. Kim et al. [22] studied how to expose a film more than 1-mm thick to obtain a precise shape. Chaudhri et al. [23] formed a 1540- $\mu$ m thick resist, but did not mention its accuracy or repeatability. They stated that the thickness of the film was controlled by the weight of the resist, but it is known that the relationship between the thickness of the film and the weight does not have high repeatability because the density of the resist is variable. Methods to simulate the conditions for high-precision exposure and development of thick SU-8 films have also been investigated [24]. Thus, studies on the exposure and development of thick films have been reported, but there are few detailed reports on how to control the film thickness through the application and soft baking process when the resist is in a liquid state that is difficult to control.

Lin et al. [25] attempted to form 500- $\mu$ m thick films using the SU-8 2000 series, and reported a formed film thickness accuracy of approximately $3 \%$. Mata et al. [26] showed the relationship between the spin coating speed of SU-8 2100 and the thickness of the applied film. When the speed was decreased, the mean film thickness and the thickness variation became large. At the lowest experimental speed of $1000 \mathrm{rpm}$, a large variation of approximately $100 \mu \mathrm{m}$ to the mean thickness of $226 \mu \mathrm{m}$ was generated.

Currently, the type of SU-8 that is readily available is of the 3000 series and not the 2000 series, and the compositions and characteristics of these two types of SU-8 differ. We attempted to form films thicker than $100 \mu \mathrm{m}$ with the SU-8 3000 series, and the results of the trials we conducted indicate that an SU-8 resist that is thickly applied on a substrate is biased during the soft baking process, and the thickness of the formed film does not become uniform, as shown in more detail later. The applied resist easily contains bubbles and generates edge beads [27, 28]. This problem becomes more serious when forming thicker films.

As a different method to form an SU-8 film, Bernasconi et al. [29] used inkjet printing. The thicknesses of the resist films made by them were on the order of $1-10 \mu \mathrm{m}$. When a film with several hundred micrometer thickness is formed using their method, issues regarding accuracy, required time, and cost will arise. Therefore, at present, it is more advantageous to improve the conventional method to form thick films. Another option is SU-8 dry film [30]. In this case, the user's choices are limited and the interface between the layers in the film may affect the product when several dry films are laminated.

The characteristics of thin films less than $10 \mu \mathrm{m}$ have been investigated by Vinje et al. [31]. They found that even in the case of thin films, the relationship between the film thickness and the spin coating speed has several variations. Not only for thick films, but also for thicknesses of $100 \mu \mathrm{m}$ or less, a method to achieve high precision and high reproducibility during the coating and soft baking processes has not been sufficiently established. This is a major problem, especially for manufacturers who massproduce SU-8 products.

The objective of this study is to establish a new method for forming uniform, thick photoresist films with a controlled thickness (set to $400 \mu \mathrm{m}$ ) that span several hundred micrometers, and thus exceed the adaptable SU-8 range. We propose a method to provide remediation of the resist film before exposure. To suitably remedy the film, soft baking before remediation is very important, but this becomes difficult with increasing film thickness. In this paper, techniques to complete soft baking are introduced. Our proposed method does not depend on the state of the film after application, so it is expected that it can be used to form not only precise, very thick SU-8 films, but also precise thin films.

\section{General SU-8 formation process and issues associated with thick films}

\subsection{General formation process for SU-8 films}

This study aimed to form a photoresist film with a uniform thickness of $400 \mu \mathrm{m}$ using the SU-8 3000 series, which is commonly used for resist films with thicknesses $\leq 100 \mu \mathrm{m}$ and which is easy to treat. The general formation procedure is summarized in Fig. 1. The remediation is an 


\section{Treatment of substrate surface}

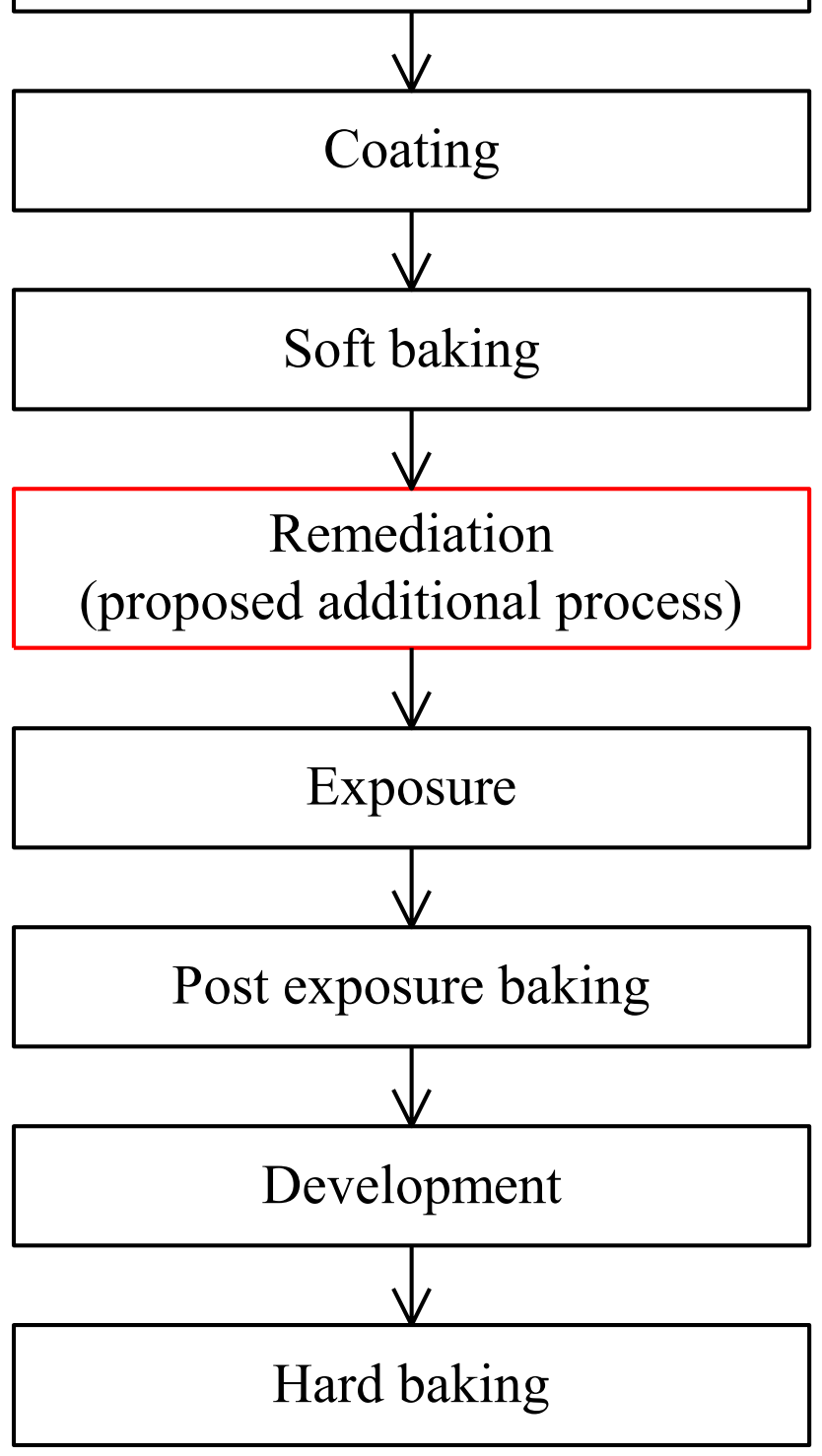

Fig. 1 Procedure to form SU-8 films

additional process proposed in this study and is not conducted in the general procedure.

At first, the surface of a substrate (typically, a mirror silicon wafer) is cleaned and treated. The required amount of photoresist is coated on the substrate. The resist coated on the substrate is heated to $95^{\circ} \mathrm{C}$ to evaporate the solvent included in the resist. A photomask with the required shape is attached to the resist surface, which is then exposed to UV rays. SU-8 is a negative-type photoresist, and its exposure to UV radiation initiates the process of crosslinking and solidification of the resist. The exposed resist is heated to $95{ }^{\circ} \mathrm{C}$ to accelerate the crosslinking process. Using a dedicated developer, unnecessary (i.e.,

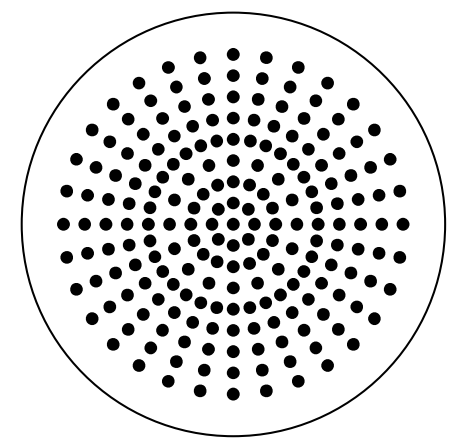

Fig. 2 Pattern formed to measure the film thickness distribution

unexposed) resist is dissolved and removed. Finally, the imaged resist is heated at $150-200{ }^{\circ} \mathrm{C}$ to complete crosslinking.

Since the soft-baked resist solidifies at a temperature below the glass transition temperature of the soft-baked SU-8, approximately $60^{\circ} \mathrm{C}$ [32], the coating and soft baking process have a significant effect on the thickness of the formed resist film. A commonly used coating method is spin coating. The relevant conditions associated with this method, such as the time sequence of the revolution speed, govern the amount of the coated resist, which determines the formed film thickness. The relationship between the spin coating conditions and the film thickness allows control of the film thickness; however, as the film becomes thicker, its reproducibility is reduced and it is difficult to control the film thickness.

\subsection{Measurement of the thickness distribution}

In this study, 4-inch mirror silicon wafers of $102 \mathrm{~mm}$ diameter were used as substrates. To evaluate the film thickness distribution, the photoresist was formed and in the shape shown in Fig. 2. Circles with diameters of $3 \mathrm{~mm}$ were arranged every $5 \mathrm{~mm}$ in radial directions within $40 \mathrm{~mm}$ from the center. Measurements in the outermost region were excluded because the thickness in this region varies significantly due to the influences of the substrate edges. After film formation, the height difference between the top of each circle and the substrate surface was measured using an electric dial gauge (ID-H0530, Mitsutoyo Corp.), the resolution of which was $0.5 \mu \mathrm{m}$. The height variation in each circle is less than $1 \mu \mathrm{m}$ and is negligible.

\subsection{Issues related to the formation of thick films}

Following the general procedure, approximately $4.5 \mathrm{~g}$ of SU-8, equivalent to approximately $400 \mu \mathrm{m}$ in thickness, was applied on a substrate and cured. The surface of the formed film became approximately flat but was 
not parallel to the substrate surface, i.e., the film thickness was biased in a certain direction as shown in Fig. 3 .

To avoid this thickness bias, several coating methods, including spin coating and spray coating, were utilized in trials. In the case of spray coating, the coating and soft baking processes were carried out by an outside contractor. However, the formed films exhibited thickness biases regardless of the coating methods, which suggests that this characteristic has a certain extent of universality. Furthermore, the degree of the bias was unrelated to the coating conditions. These results indicate that the film thickness bias can be attributed to the soft baking process and not to the coating process.

The applied resists were soft-baked on a precisely leveled hot plate (the horizontal levelness was less than $0.1 \mathrm{~mm} / \mathrm{m}$ ) or an intentionally tilted hot plate (the horizontal levelness was $100 \mathrm{~mm} / \mathrm{m}$ ) on a trial basis. However, both the levelness and direction correlations between the inclination of the hot plate and the thickness bias of the soft-baked resist film were not confirmed. Thus, the inclination of the hot plate is not the cause of the film bias. In this study, no special care was taken to decrease the amount of vibration during soft baking; thus, slight vibration may be one of the causes of the bias in the film thickness. Nevertheless, it is difficult to prevent this bias from occurring during soft baking when considering other factors, such as the non-uniformity after application.

When the required film thickness is in the normally adaptable range for each type of SU-8 according to the manufacturing specifications, the weight of the coated resist governed by the spin coating condition corresponds to the formed film thickness. However, the thickness cannot be controlled using the coated resist weight or the spin coating conditions for thick films because the relationship between the resist weight and the formed film thickness exhibits low repeatability, as explained in a subsequent section.

Because of these specific problems related to thick films, a remediation method that can eliminate the bias of the soft-baked resist and set the film thickness to a desired value is considered practical.

\section{Remediation method}

\subsection{Concept of the remediation method}

Figure 4 shows a schematic diagram of the proposed photoresist film remediation method. The remediation equipment is self-made and consists of a base and a cover made of precision-machined stainless steel plates. Residual
Fig. 3 Typical thickness distribution of thick photoresist films formed using the general process. In this case, $x$ denotes the film thickness bias direction. The original point is located at the center of the substrate. The red line indicates the approximation curve of the results excluding the values that have a tendency to be smaller near the periphery
Fig. 4 Schematic diagram of the remediation process
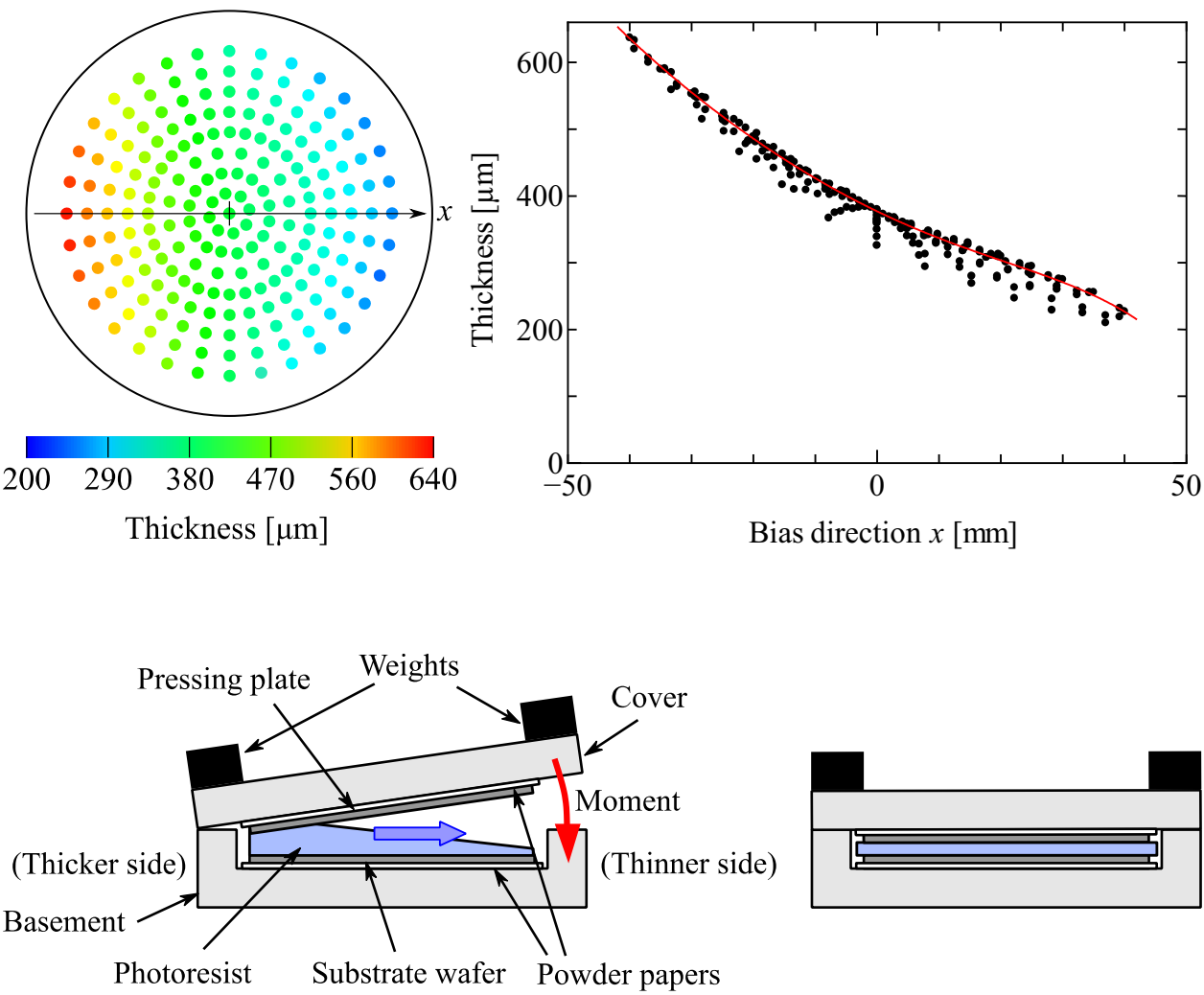

Photoresist

During remediation 
stresses in the plates were eliminated with careful heat treatment. The cover is flat, and the basement has a uniform gap and the bottom of the very shallow and wide square groove is as flat as the cover. A silicon wafer identical to the substrate is used as a pressing plate and is placed in contact with the photoresist. To easily remove the pressing plate from the resist after remediation, a mold release agent, comprised primarily of trimethylbenzene, xylene, and paraffin, is applied on the pressing plate surface that is polished until a mirror-like surface is obtained. To prevent adhesion of the resist that is pushed away from the substrate to the remediation equipment, common powder papers are inserted between the base and the substrate and between the cover and the pressing plate, respectively. The papers also allow the quick removal of the substrate from the remediation equipment after remediation. Because the soft-baked photoresist is solid and cannot be easily deformed at temperatures below the glass transition temperature, the equipment is heated above the glass transition temperature in a convection oven in advance. The heated resist thus fluidizes during remediation.

The pressing plate was lowered onto the soft-baked resist, and the bias direction of the resist thickness was confirmed by measuring the variation in height between the bottom of the substrate and the top of the pressing plate (i.e., the variation in the resist thickness) along the outer circumference of the substrate. A dial gauge was used to conduct this measurement, and the variation in the measured thickness was of the order of 10-100 $\mu \mathrm{m}$. The substrate is placed on the paper in the gap of the base. The direction of the ends of the base and that of the resist thickness bias are aligned. The pressing plate, another paper, and the cover are then piled on the resist. Some weights are loaded onto the cover, keeping the thicker-side ends of the base and the cover in contact, and enabling the thinner-side end of the cover to float. The weights induce a moment around the thicker-side end and deform the resist. The excess resist on the thicker side is, therefore, pushed into the thinner side. As the remediation progresses, the thinner-side end of the cover falls down. When both ends of the cover are in contact with the base ends, the bottom of the base gap and the cover become parallel. The thickness uniformities of the silicon wafers and the powder papers were confirmed by measurements. Accordingly, this ensures that the gap between the two silicon wafers is uniform, i.e., the thickness of the remedied film becomes uniform and equal to the value obtained by subtracting the thickness of the silicon wafers and the powder papers from the depth of the base gap.

During remediation, some resist is pushed out from the substrate edge. The resist initially coated on the substrate must include this extruded resist, which is easily removed after remediation. In order to obtain an appropriately remedied film, the points mentioned in the following sections should be considered.

\subsection{Soft baking}

The extra photoresist pushed out from the substrate during remediation should be included in the resist coated on the substrate. Approximately $6 \mathrm{~g}$ of applied resist is required to form an SU-8 with a film thickness of $400 \mu \mathrm{m}$ on a 4-inch silicon wafer. If this quantity of SU-8 is softbaked by normal heating, some or all of the resist sticks to the pressing plate during remediation, and the film is damaged when the pressing plate is removed after remediation, as shown in Fig. 5. The broken resist remains sticky and solvent-rich because soft baking is not completed before remediation.

When the resist is heated on a hot plate, the solvent evaporates from the resist surface and the solvent concentration on the surface is decreased relative to that in the bulk. When the resist surface is sufficiently dried, the solvent-rich resist in the bulk usually does not affect the film formation when the conventional method is performed. However, if remediation is conducted before exposure, the solvent-rich resist is deformed, and then it can come into contact with and adhere to the pressing plate. To prevent this adhesion, the solvent concentration in the entire resist should be sufficiently reduced before remediation.

Because the bias of the resist thickness is induced during the soft baking process and the degree of the bias is not reproducible, the required time to complete solvent evaporation from the thick side of the resist varies depending on the degree of bias. In addition, the surface resists that dries early in the heating process forms a skin that

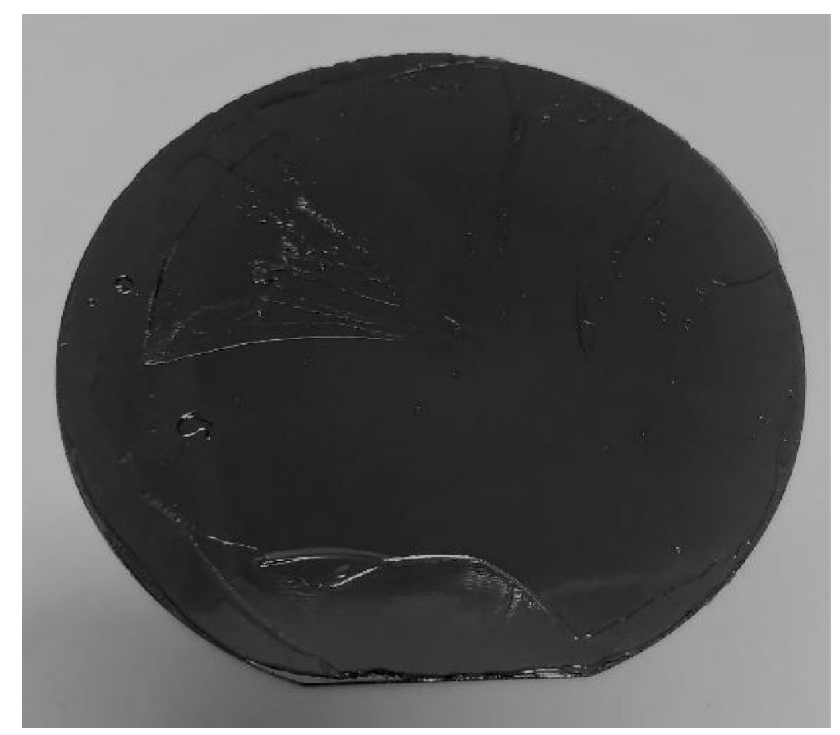

Fig. 5 Damaged photoresist film after remediation 
prevents solvent evaporation from the bulk resist. Thus, sufficient drying of the bulk resist cannot be achieved by simply extending the heating time. In some cases, soft baking for a long duration ( $>1$ day) is insufficient.

The outcomes of trial and error experiments revealed that the following methods could solve this problem and prevent adhesion of the resist to the pressing plate during remediation.

\subsubsection{Decreasing the solvent concentration of the initial photoresist}

The solvent included in SU-8 before use evaporates at room temperature. After breaking the seal of an SU- 8 bottle, the solvent concentration decreases continuously. Old resist stock (stored in bottles that were opened previously) can be easily soft-baked.

The solvent concentration of the resist (in other words, the density of the resist) before use is not constant because of the time-dependent evaporation of the solvent. This means that the film thickness cannot be controlled by the spin coating condition and/or the weight of the resist coated on the substrate. Therefore, the remediation method, which allows control of the film thickness regardless of the resist weight, is highly beneficial.

\subsubsection{Soft baking temperature and time}

The standard soft baking temperature is $95^{\circ} \mathrm{C}$. Johari et al. [33] stated that $85^{\circ} \mathrm{C}$ is suitable to soft-bake SU-8 2010 film with a thickness of $30 \mu \mathrm{m}$; however, we confirmed that soft baking of SU-8 3025 at higher temperatures is possible. The results of various trials show that soft baking at higher temperatures increases the solvent evaporation rate, but excessively high temperatures and/or excessively long heating make the resist too hard to deform, as well as insoluble in the developer, so that the maximum temperature to soft-bake is $140{ }^{\circ} \mathrm{C}$. The heating time at the maximum temperature should be limited to $2 \mathrm{~h}$. All the solvent should evaporate from the resist under these conditions.

\subsubsection{Multiple coating and soft baking}

Decreasing the ratio of the bulk volume to the surface area of the resist decreases the solvent concentration distribution in the soft-baked resist. The surface area of the resist is equivalent to that of the substrate and cannot be varied, so the decreased resist volume contributes to the uniformity of the solvent concentration. If the required amount of resist is divided into multiple amounts and the divided resists are coated and soft-baked repeatedly, the solvent concentration in the soft-baked resist layers accumulated on the substrate can become almost uniform.
For additional resist coated on a soft-baked resist, the solvent in the additional resist not only evaporates from the new surface but also penetrates the dried layer beneath it. Therefore, it is difficult to evaporate the solvent from additional resist. It is more effective to reduce the amount of resist in the new layer than to divide the total amount equally.

An increased number of divisions decreases the distribution of the solvent concentration. However, if the number of divisions is excessive, the cumulative heating time of the first resist coating will exceed the limit $(2 \mathrm{~h})$. The appropriate number of divisions for the formation of a film with a thickness of $400 \mu \mathrm{m}$ is in the range of 2-4.

\subsubsection{Convection flow mixing}

By shifting the photoresist mechanically during the soft baking process, convective flows are generated in the resist. To generate these types of flows, two methods, one in which the substrate is vibrated and the other in which a needle inserted into the resist is moved, are adopted. By conducting these methods in parallel, the solvent concentration in the resist can be equalized. The needle also breaks the skin formed on the resist surface and facilitates solvent evaporation from the bulk.

It should be noted that the net evaporation rate is decreased if convective flow unnecessarily mixes the topmost resist layer with the dried layer underneath it. The flow induced by the needle should be restricted to the topmost layer, as shown in Fig. 6. The resist has high viscosity and the generated flow is laminar, so it is not expected that mixing will cause turbulent vortices. Accordingly, the proposed methods are not fully effective, but they are still necessary. If a more effective convective flow method is devised, soft baking could become easier and more certain.

\subsubsection{SU-8 product variety}

The currently available commercial SU-8 3000 series includes a variety of products with different viscosities. Low-viscosity resist includes more solvent and thus

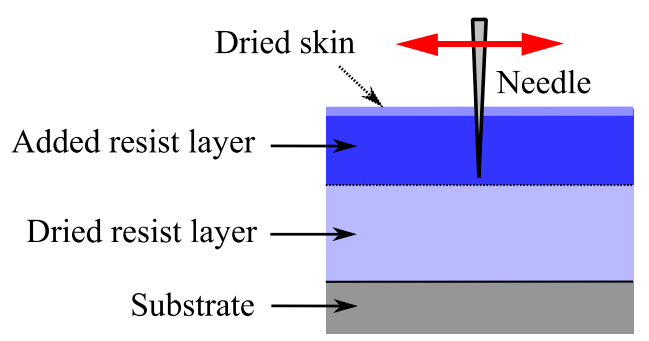

Fig. 6 Mixing the added resist layer by a needle 
requires a longer time for soft baking. For an excessively low viscosity, retaining the required amount of resist on the substrate until the soft baking process is completed is difficult, because the resist spills from the substrate under its own weight. Conversely, with high-viscosity resist, convective flow for mixing is not easily enabled. For the conditions investigated in this study, SU-8 3025 is the most suitable because its viscosity is in the intermediate range.

By combining the methods and conditions mentioned above, the solvent concentration in the entire resist can be reduced to allow remediation of the resist for films with a thickness of $400 \mu \mathrm{m}$. For films with a thickness $>400 \mu \mathrm{m}$, soft baking the resist to allow remediation using the presented methods would be difficult. Improvement of the soft baking method can enhance this remediation method and make it applicable to thicker films.

\subsection{Remediation conditions}

Because the glass transition temperature of the softbaked SU-8 is approximately $60^{\circ} \mathrm{C}$, the remediation equipment is heated to $90^{\circ} \mathrm{C}$ in advance and is kept warm in an oven during remediation. The heated photoresist fluidizes and is remedied by the moment applied to the cover. The excess resist on the thicker side is forced to the thinner side to compensate. Simultaneously, some of the excess resist is pushed out from the substrate edge. After filling the thinner side, all excess resist is extruded as the remediation is completed.

A larger moment shortens the time required for the remediation process. However, an excessive moment induces excessive extrusion of the resist from the thicker-side edge and prevents filling of the thinner side. In this case, the thin region remains after remediation, as shown in Fig. 7. However, if the moment is too small and the remediation time is excessive, the resist continues to leak from all surrounding edges not only because of the loaded moment but also because of the weight of the resist. As a result, the film becomes thinner at the outer edge of the substrate, as shown in Fig. 8. Thus, it is preferable to minimize the remediation time. In this study, the cover weighed $2 \mathrm{~kg}$, and the weights of the mounted on the cover weighed 3-14 kg in total. By changing the weights and their positions on the cover, the loaded moment at the contact point between the basement
Fig. 7 Instance of thickness distribution of a photoresist remedied with excessive force. The red line indicates the approximation curve of the results
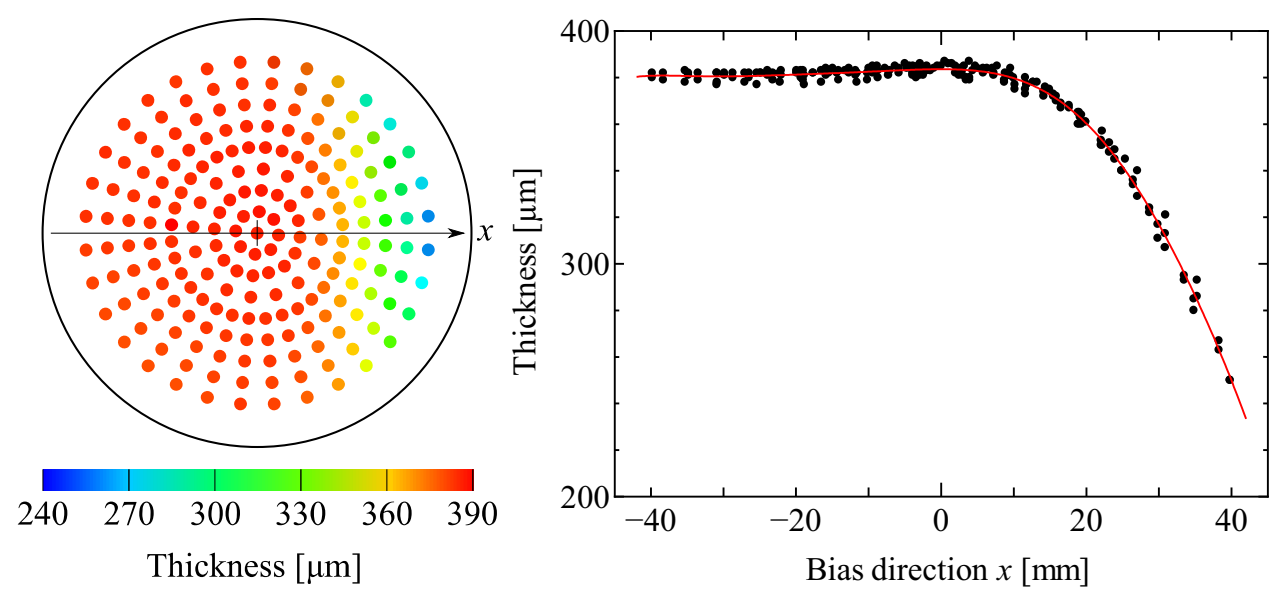

Fig. 8 Instance of thickness distribution of a photoresist film with excessive and prolonged heated remediation. The red line indicates the approximation curve of the results
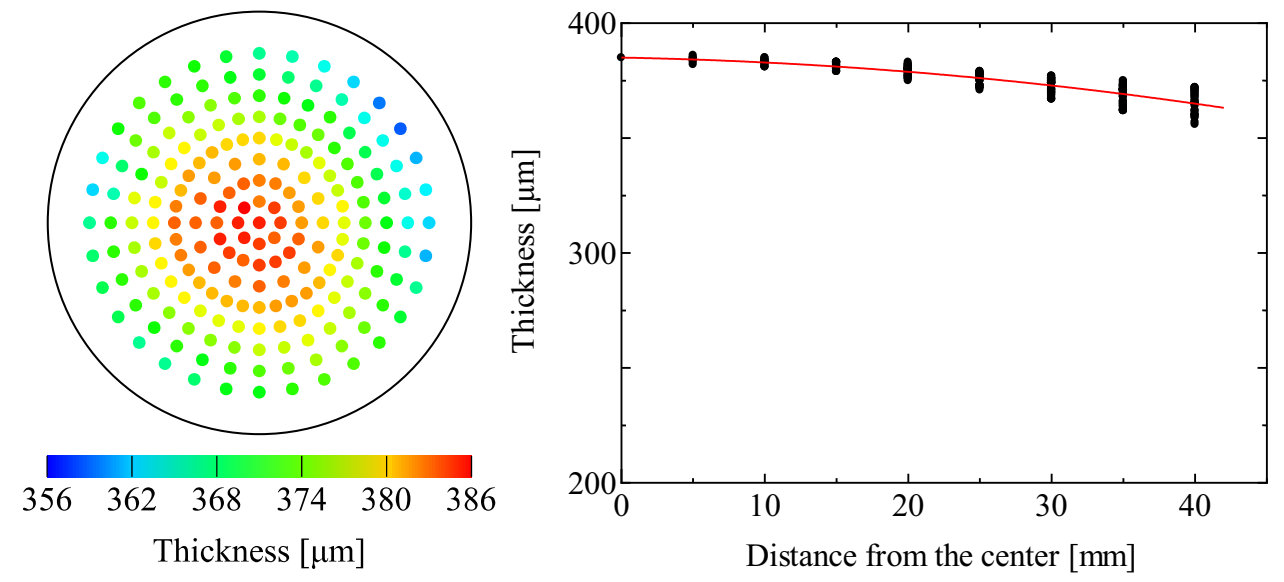
and the cover was varied between 3 and $11 \mathrm{Nm}$ that is the suitable condition loading the cover.

An appropriate moment should be loaded on the cover to complete the remediation in the shortest possible period, while fully supplying resist to the thinner side. It is difficult to determine the appropriate moment quantitatively because the bias degree of the soft-baked resist is not reproducible. The moment must be adjusted based on the bias degree. As a guide, a proper film can be obtained if the remediation is completed within approximately $10 \mathrm{~min}$.

After remediation, the resist should be immediately cooled to below the glass transition temperature of softbaked SU-8 to stop unexpected resist leakage. The remediation equipment requires several hours to cool naturally because it possesses a large heat capacity. Forced cooling may induce distortion of the equipment owing to thermal shrinkage, and if this happens, the equipment becomes useless. Therefore, the remediated resist on the substrate must be removed immediately after remediation. The weights and the cover should be removed first, and then, the ends of the powder papers that sandwich the resist on the substrate and the pressing plate should be pinched and drawn out. At that moment, force must not be applied to the pressing plate because the hot resist can be easily deformed. The removed resist cools immediately because of its small heat capacity.

The pressing plate is removed from the cooled and solidified resist. As the resist may remain somewhat sticky, removal while cooling the pressing plate effectively prevents adhesion of the resist to the pressing plate. However, excessive cooling induces cracks in the resist owing to thermal shrinkage; thus, the minimum cooling temperature should be $-10^{\circ} \mathrm{C}$.

The optimal conditions described thus far to obtain a remedied SU-8 film with a thickness of $400 \mu \mathrm{m}$ on a substrate comprising a 4-inch silicon wafer are summarized in Table 1.

Table 1 Optimal conditions to obtain a remedied SU-8 with a thickness of $400 \mu \mathrm{m}$ on a substrate comprising a 4-inch silicon wafer

\begin{tabular}{ll}
\hline Photoresist type & $\mathrm{SU}-83025$ \\
Amount of applied photoresist & $6 \mathrm{~g}$ \\
$\begin{array}{l}\text { Number of times to divide the application and soft bak- } \\
\text { ing process }\end{array}$ & 4 \\
$\begin{array}{l}\text { Soft baking temperature } \\
\text { Heating time limit for soft baking }\end{array}$ & $140^{\circ} \mathrm{C}$ \\
Heating temperature during remediation & $2 \mathrm{~h}$ \\
$\begin{array}{l}\text { Loaded moment to remedy the resist } \\
\text { Average remediation time }\end{array}$ & $90{ }^{\circ} \mathrm{C}$ \\
$\begin{array}{l}\text { Cooling temperature when removing the pressing } \\
\text { plate }\end{array}$ & $3-11 \mathrm{Nm}$ \\
\hline
\end{tabular}

The subsequent procedure after remediation is the same as that followed in the general method, although the exposure and development periods should be longer according to the film thickness.

\section{Remediation result}

An edge image of an SU-8 pattern formed through the proposed procedure was captured using an optical microscope, as shown in Fig. 9. This image was captured by tilting the substrate, and the shadow indicates the sidewalls of the pattern. The lens is focused on the top corner of the formed resist pattern. In contrast, the tangency of the bottom of the pattern to the base of the pattern appears blurred and out of the depth of field. This image difference between the top and bottom of the pattern indicates the distance between them that is the height of the pattern. In this study, the formation processes before the exposure were the focus, and no special treatment was conducted in the exposure and development processes. Nonetheless, a sharp edge was obtained and it confirmed that the proposed procedure does not affect the exposure and development processes. As the image is only partially in focus, it verifies that the pattern is very tall. The surface shape of the remedied photoresist is copied from that of the pressing plate. Because a mirror-finish silicon wafer is used as a pressing plate, the surface of the remedied resist also has a mirror finish.

Figure 10 shows an example of the thickness distribution of an SU-8 film that was remedied using the proposed procedure. Because some photoresist leaks from the substrate edge during heating remediation, the film becomes slightly thinner at the outer edge of the substrate. However, this tendency is minimized and is not observed in the center region, which has a diameter equal to $40 \mathrm{~mm}$. Here after, the mean value of the thickness in this center region is defined as the mean thickness. The largest

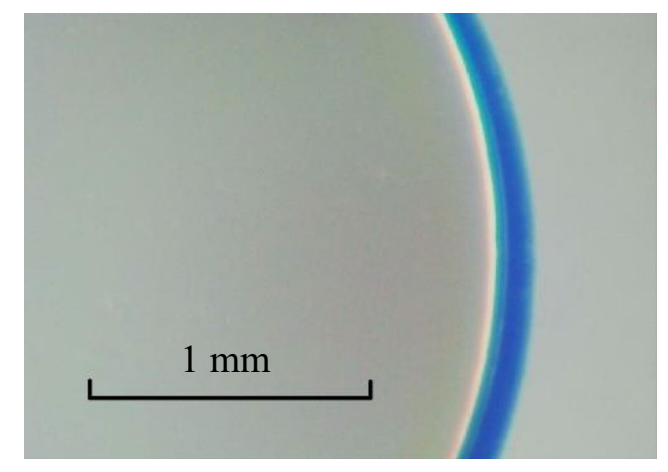

Fig. 9 Formed pattern edge image captured from an oblique direction 


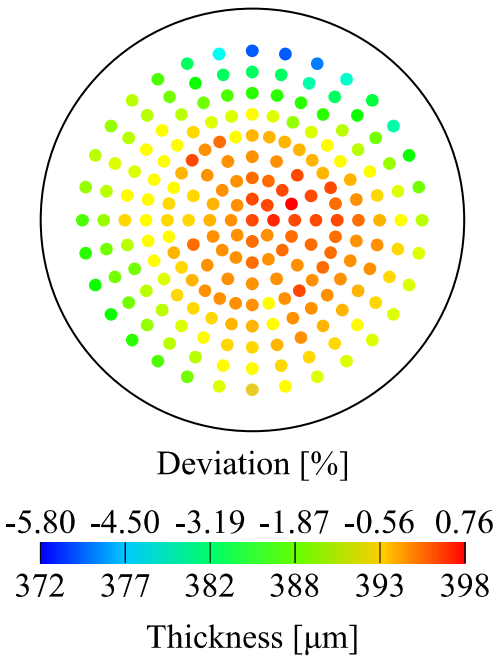

Fig. 10 Example of thickness distribution of photoresist layers remedied by the optimal procedure

deviation of the local thickness from the mean thickness at the outer side is $-5 \%$. The deviation in the center region is within $\pm 1 \%$. Even when the entire area is considered, the proposed method guarantees sufficiently higher accuracy compared to that formed by the conventional method, and considering the dimensions of practical micro channels, the precision of the remedied film is sufficient for use.

Ideally, an appropriately remedied film has a thickness identical to the value obtained by subtracting the thicknesses of the two silicon wafers (the substrate and pressing wafer) and the two powder papers from the depth of the base gap. The thickness of the paper is $30 \mu \mathrm{m}$ and exhibits little variation. The silicon wafer has a thickness tolerance of $\pm 25 \mu \mathrm{m}$, which influences the thickness of the remedied resist film. Figure 11 shows the relationship between the sum of the thicknesses of the two wafers and the mean thickness of the film. The straight line is the fitted line, which has a slope of -1 . The intercept value on the vertical axis is $1444 \mu \mathrm{m}$, which can be regarded as the substantial value obtained by subtracting the thicknesses of the two papers from the gap depth of the base. The deviation of the film mean thickness from the approximation line is $\pm 4 \mu \mathrm{m}$, i.e., $\pm 1 \%$. The remedied film thickness can thus be controlled by the wafer thicknesses with high precision. In addition, it is practical to vary the film thickness by changing the paper thickness and the gap depth.

In this study, the target thickness of the formed film was set to $400 \mu \mathrm{m}$. In thinner cases than $400 \mu \mathrm{m}$, the soft baking process becomes easier and the film thickness can be reliably controlled by changing the gap depth of the base of the remediation equipment. Furthermore, by making a difference in the height at both ends of the base and remedying a film with tilting cover, it is

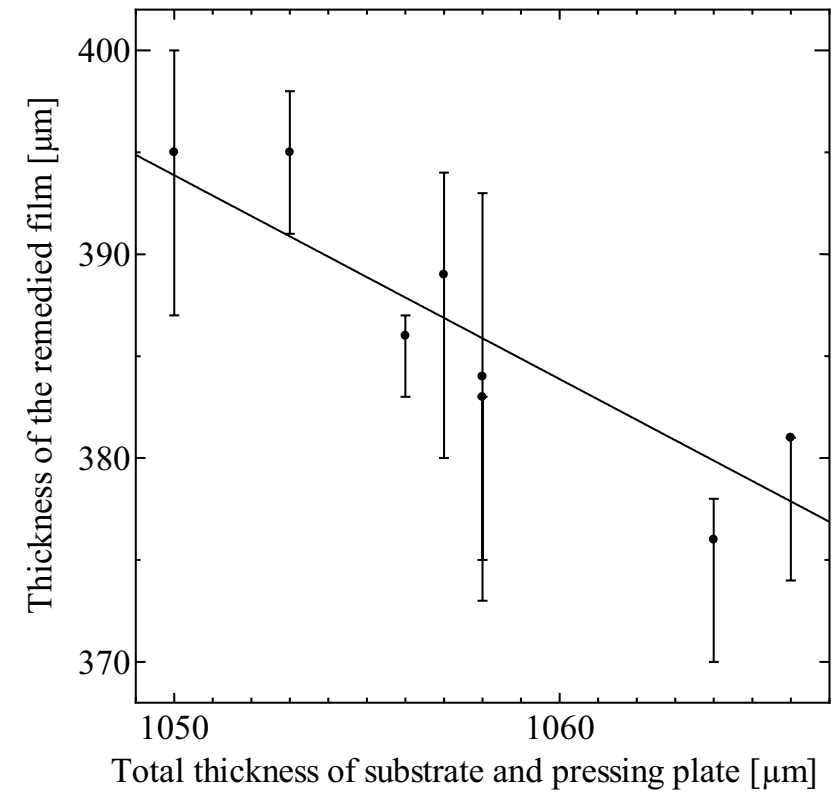

Fig. 11 Relationship between the mean thickness of the remedied photoresist film and the total thickness of the two silicon wafers of the substrate and pressing plate. The marks and error bars indicate the mean values and the deviations in the local values, respectively. The slope of the approximation line is -1

possible to gradually change the thickness of the film. It is also possible to form resist films of uniform thickness on substrates that are larger than 4-inch mirror silicon wafers.

\section{Conclusions}

In order to realize the facile and reliable production of micro channels with depths spanning several hundred micrometers, we developed a technique to form photoresist films with uniform high thicknesses by enhancing the SU-8 processing range. Assuming a target thickness of $400 \mu \mathrm{m}$ and a 4-inch silicon wafer substrate, the following conclusions are obtained.

(1) If the required amount of SU-8 is formed using conventional methods, the film thickness is not well controlled and is directionally biased.

(2) A uniform film with the desired thickness can be obtained if the soft-baked resist is appropriately remedied before exposure.

(3) To prevent adhesion of the resist to the pressing plate during remediation, the solvent concentration in the entire resist should be reduced to an appropriate level by soft baking. Several methods are proposed for solvent concentration reduction. 
(4) An appropriate load imposed on the cover during remediation and quick cooling of the resist after remediation yields suitable remediation results.

(5) The accuracy of the remedied film thickness in the center region of $40 \mathrm{~mm}$ in diameter is within $\pm 1 \%$ and the largest deviation across the entire area is $-5 \%$. These films are acceptable for practical uses.

(6) The remedied film thickness is calculated by subtracting the thicknesses of the substrate, the pressing plate, and two powder papers from the gap depth of the base. The film thickness can be controlled by manipulating these parameters.

\section{Compliance with ethical standards}

Conflicts of interest The authors that they have no conflict of interest

\section{References}

1. Xia Y, Whitesides GM (1998) Soft lithography. Annu Rev Mater Res 28:153-184

2. McDonald JC, Whitesides GM (2002) Poly(dimethylsiloxane) as a material for fabricating microfluidic devices. Acc Chem Res 35:491-499

3. Lim CT, Low HY, Ng JKK, Liu WT, Zhang Y (2009) Fabrication of three-dimensional hemispherical structures using photolithography. Microfluid Nanofluidics 7:721-726

4. Fujiwara T, Johnston ID, Tracey MC, Tan CKL (2010) Increasing pumping efficiency in a micro throttle pump by enhancing displacement amplification in an elastomeric substrate. J Micromech Microeng 20:065018

5. Johnston ID, McCluskey DK, Tan CKL, Tracey MC (2014) Mechanical characterization of bulk Sylgard 184 for microfluidics and microengineering. J Micromech Microeng 24:035017

6. Matsumoto S, Leclerc E, Maekawa T, Kinoshita H, Shinohara M, Komori K, Sakai Y, Fujii T (2018) Integration of an oxygen sensor into a polydymethylsiloxane hepatic culture device for twodimensional gradient characterization. Sens Actuators B Chem 273:1062-1069

7. Weilin Q, Mudawar I (2002) Experimental and numerical study of pressure drop and heat transfer in a single-phase micro-channel heat sink. Int J Heat Mass Transf 45:2549-2565

8. Fujiwara $\mathrm{T}$, Ohue $\mathrm{H}$, Ushijima T, Kitoh O (2007) Optimization of channel geometry and pumping conditions for an alternate pumping microreactor. J Fluid Sci Technol 2:389

9. Takahashi Y, Okajima J, Iga Y, Komiya A, Maruyama S (2014) Preliminary experiment of supersonic micro-channel gas flow visualization by using Interferometer. J Fluid Sci Technol. https ://doi.org/10.1299/jfst.2014jfst0069

10. Nagai M, Tanizaki K, Shibata T (2019) Batch assembly of SU-8 movable components in channel under mild conditions for dynamic microsystems: application to biohybrid systems. J Microelectromech Syst 28:419

11. Yang Y, Lai L, Ding G, Chen T (2019) SiC nanowire-based SU-8 with enhanced mechanical properties for MEMS structural layer design. Nanotechnol Precis Eng 2:169-176
12. LaBianca NC, Gelorme JD (1995) High-aspect-ratio resist for thick-film applications. Proc SPIE 2438:846-852

13. Lorenzdag $H$, Despont $M$, Fahrnidag N, LaBianca N, Renauddag P, Vettiger P (1997) SU-8: a low-cost negative resist for MEMS. J Micromech Microeng 7:121-124

14. Dellmann L, Roth $S$, Beuret $C$, Racine GA, Lorenz H, Despont $M$, Renaud P, Vettiger P, De Rooij NF (1998) Fabrication process of high aspect ratio elastic and SU-8 structures for piezoelectric motor applications. Sens Actuators A Phys 70:42-47

15. Stangegaard M, Wang Z, Kutter JP, Dufva M, Wolff A (2006) Whole genome expression profiling using DNA microarray for determining biocompatibility of polymeric surfaces. Mol Biosyst 2:421-428

16. Abgrall P, Conedera V, Camon H, Gue AM, Nguyen NT (2007) SU-8 as a structural material for labs-on-chips and microelectromechanical systems. Electrophoresis 28:4539-4551

17. Du L, Zhai K, Li X, Liu S, Tao Y (2020) Ultrasonic vibration used for improving interfacial adhesion strength between metal substrate and high-aspect-ratio thick SU-8 photoresist mould. Ultrasonics 103:106100

18. Mao Z, Yoshida K, Kim J (2019) Releasing large-area SU-8 structures without using any sacrificial layers. Microelectron Eng 212:53-60

19. Chuang YJ, Tseng FG, Lin WK (2002) Reduction of diffraction effect of UV exposure on SU-8 negative thick photoresist by air gap elimination. Microsyst Technol 8:308-313

20. Liu J, Cai B, Zhu J, Ding G, Zhao X, Yang C, Chen D (2004) Process research of high aspect ratio microstructure using $\mathrm{SU}-8$ resist. Microsyst Technol 10:265-268

21. Amato L, Keller SS, Heiskanen A, Dimaki M, Emnéus J, Boisen A, Tenje M (2012) Fabrication of high-aspect ratio SU-8 micropillar arrays. Microelectron Eng 98:483-487

22. Kim JJK, Thuwaini HA, Almuslem M (2018) Photolithography of SU-8 microtowers for a 100-turn, 3-D toroidal microinductor. Micro Nano Syst Lett 6:14

23. Chaudhri BP, Ceyssens F, Moor PD, Hoof CV, Puers R (2010) A high aspect ratio SU-8 fabrication technique for hollow microneedles for transdermal drug delivery and blood extraction. J Micromech Microeng 20:064006

24. Zhou Z, Huang Q (2018) Comprehensive simulations for ultraviolet lithography process of thick SU-8 photoresist. Micromachines 9:341

25. Lin C, Lee G, Chang B, Chang G (2002) A new fabrication process for ultra-thick microfluidic microstructures utilizing SU-8 photoresist. J Micromech Microeng 12:590-597

26. Mata A, Fleischman AJ, Roy S (2006) Fabrication of multi-layer SU-8 microstructures. J Micromech Microeng 16:276-284

27. Stevens J, Olgado D, Ko A, Mok YF (2003) Edge bead removal/ spin rinse dry (EBR/SRD) module. United States patent US 6516815:B1

28. Lee $\mathrm{H}$, Lee $\mathrm{K}, \mathrm{Ahn} \mathrm{B}, \mathrm{Xu} J, \mathrm{Xu} \mathrm{L}, \mathrm{Oh} \mathrm{KW}$ (2011) A new fabrication process for uniform SU-8 thick photoresist structures by simultaneously removing edge bead and air bubbles. J Micromech Microeng 21:125006

29. Bernasconi R, Costa Angeli M, Mantica F, Carniani D, Magagnin $L$ (2019) SU-8 inkjet patterning for microfabrication. Polymer $185: 121933$

30. Wangler N, Beck S, Ahrens G, Voigt A, Grützner G, Müller C, Reinecke $H$ (2012) Ultra thick epoxy-based dry-film resist for high aspect ratios. Microelectron Eng 97:92-95

31. Vinje J, Beckwith KS, Sikorski P (2020) Electron beam lithography fabrication of SU-8 polymer structures for cell studies. J Microelectromech Syst 29:160-169

32. Blanco FJ, Agirregabiria M, Garcia J, Berganzo J, Tijero M, Arroyo MT, Ruano JM, Aramburu I, Mayora K (2004) Novel three-dimensional embedded SU-8 microchannels fabricated using a low 
temperature full wafer adhesive bonding. J Micromech Microeng 14:1047

33. Johari S, Tamilchelvan N, Normf MNM, Ramli MM, Taib BN, Mazalan M, Wahab Y (2014) The effect of softbaking temperature on SU-8 photoresist performance. IEEE-ICSE2014 Proc 467-470
Publisher's Note Springer Nature remains neutral with regard to jurisdictional claims in published maps and institutional affiliations. 